\title{
ARTICLE
}

Received 27 Jan 2015 | Accepted 6 Jun 2015 | Published 4 Aug 2015

DOI: $10.1038 /$ ncomms 8756

OPEN

\section{Rare coding variants and X-linked loci associated with age at menarche}

Kathryn L. Lunetta et al."

More than 100 loci have been identified for age at menarche by genome-wide association studies; however, collectively these explain only $\sim 3 \%$ of the trait variance. Here we test two overlooked sources of variation in 192,974 European ancestry women: low-frequency proteincoding variants and $\mathrm{X}$-chromosome variants. Five missense/nonsense variants (in ALMS1/ LAMB2/TNRC6A/TACR3/PRKAG1) are associated with age at menarche (minor allele frequencies 0.08-4.6\%; effect sizes 0.08-1.25 years per allele; $P<5 \times 10^{-8}$ ). In addition, we identify common X-chromosome loci at IGSF1 (rs762080, $P=9.4 \times 10^{-13}$ ) and FAAH2 ( $r 55914101, P=4.9 \times 10^{-10}$ ). Highlighted genes implicate cellular energy homeostasis, posttranscriptional gene silencing and fatty-acid amide signalling. A frequently reported mutation in TACR3 for idiopathic hypogonatrophic hypogonadism (p.W275X) is associated with 1.25year-later menarche $\left(P=2.8 \times 10^{-11}\right)$, illustrating the utility of population studies to estimate the penetrance of reportedly pathogenic mutations. Collectively, these novel variants explain $\sim 0.5 \%$ variance, indicating that these overlooked sources of variation do not substantially explain the 'missing heritability' of this complex trait. 
A ge at menarche, the onset of first menstruation in females, indicates the start of reproductive maturity and is a commonly reported marker of pubertal timing. One hundred and six genomic loci for this highly heritable trait have been mapped by genome-wide association studies (GWAS), implicating many previously unsuspected mechanisms ${ }^{1}$. However, to date that approach has been limited to consideration of only those genetic variants captured by autosomal HapMap2 reference panels. In particular, like most GWAS for other complex traits, previous GWAS for age at menarche provided poor coverage for low-frequency variants and omitted sex chromosome data.

Here we report a dual strategy for assessing genetic variation overlooked by those prior efforts: low-frequency protein-coding variants genotyped by large-scale exome-focussed arrays and high-density X-chromosome single-nucleotide polymorphism (SNP) genotyping and imputation. We identify several new associations between rare protein-coding and X-linked variants with age at menarche in women of European ancestry. The findings implicate new mechanisms that regulate puberty timing, but collectively these novel variants explained only $\sim 0.5 \%$ of the variance, indicating that these often overlooked sources of variation that do not substantially explain the 'missing heritability' of this complex trait.

\section{Results}

From the exome array studies, 61,734 low-frequency (minor allele frequency $(\mathrm{MAF})<5 \%$ ) variants passed quality-control (QC) criteria in a combined sample of up to 76,657 women of European ancestry from 19 studies (Supplementary Table 1). Gene-based burden and SKAT tests that aggregate the effects of variants with $\mathrm{MAF}<1 \%$ yielded no significant associations with age at menarche. A linear regression test was used to derive all $P$ values obtained in this study. Meta-analysis of individual variant associations with questionnaire-reported variation in age at menarche (restricted to the ages of 9-17 years) in this discovery phase identified one signal at genome-wide statistical significance $\left(P<5 \times 10^{-8}\right)$; this was a rare missense variant in the Alström's syndrome gene (ALMS1, rs45501594, p.T3544S, MAF 1\%; $\left.P=4.6 \times 10^{-10}\right)$. For follow-up testing in up to 116,317 independent women of European ancestry from the deCODE (Diabetes Epidemiology: Collaborative analysis of Diagnostic criteria in Europe ) and 23andMe studies, we selected rs45501594 and 23 other variants that met the following criteria: protein coding, present in over half of the exome array studies, and with association $P<5 \times 10^{-4}$. In the follow-up samples, 7 of the 20 variants that passed QC showed directionally concordant confirmatory associations with $P<0.05$, of which five reached genome-wide significance in a combined meta-analysis of discovery phase and follow-up data (Table 1, Fig. 1, Supplementary Fig. 1). No significant heterogeneity between studies was observed at any of these loci (Supplementary Fig. 2).

The rare missense variant in ALMS1 (rs45501594, Supplementary Fig. 3) remained the strongest signal identified using exome array studies (combined: $P=6.8 \times 10^{-20}$ ). In the follow-up samples, each rare allele was associated with 0.23 -yearlater age at menarche, an effect size more than double that of any genetic variant previously reported for puberty timing in the general population. This strong signal was not detected by the previous HapMap2-based GWAS as it is poorly tagged by common SNPs in that reference panel (maximum proxy SNP, $\left.r^{2}=0.24\right)$. Deleterious mutations in this gene cause Alström's syndrome (OMIM no. 203800), a rare, autosomal-recessive disorder characterized by cone-rod dystrophy, sensorineural hearing loss, dilated cardiomyopathy, childhood obesity, insulin resistance, diabetes mellitus, hypogonadotropic hypogonadism in males, menstrual irregularities and early puberty in females, and short stature in adulthood ${ }^{2}$. Hypogonadism was also invariably observed in an ALMS1 gene-trapped mouse model ${ }^{3}$.

The variant with largest effect was a rare stop-gain mutation in the tachykinin receptor 3 gene (TACR3; rs144292455, $\mathrm{MAF}=0.08 \%$, combined $P=2.8 \times 10^{-11}$, Supplementary Fig. 3); in follow-up samples each rare allele was associated with 1.25year-later age at menarche. Common HapMap2 SNPs at the TACR3 locus were previously associated with age at menarche ${ }^{1}$; however, the rare variant rs 144292455 is not tagged by the HapMap2 or conventional $1000 \mathrm{G}$ imputation (it was directly genotyped in 23andMe and was imputed in deCODE). Statistical independence was confirmed by observing significant association with the common TACR3 SNP in a sensitivity analysis within a participating study (Women's Genome Health Study (WGHS), Supplementary Table 1) that excluded rare allele carriers. The rare allele causes a premature stop codon (p.W275X) in the fifth transmembrane segment of the 465 amino-acid receptor for the neuropeptide neurokinin $\mathrm{B}$, and is the most frequently reported TACR3 mutation in the rare reproductive disorder idiopathic hypogonadotropic hypogonadism (idiopathic hypogonadotropic hypogonadism (IHH), OMIM no. 614840) ${ }^{4}$. Both homozygous and heterozygous p.W275X variants have been reported in male $\mathrm{IHH}$ cases with features of 'early androgen deficiency'; however, notably the heterozygous cases showed evidence of spontaneous neuroendocrine recovery. Our findings suggest that heterozygous p.W275X variants contribute to the normal variation in puberty timing, whereas homozygous inheritance or possibly compound heterozygosity is required for $\mathrm{IHH}$.

A low-frequency missense variant in the $L A M B 2$ gene was associated with 0.08-year-later age at menarche (rs35713889, p.G914R, MAF $4 \%$; $P=1.1 \times 10^{-11}$; Supplementary Fig. 3). In the same region (3p21.31) we previously reported a HapMap2 GWAS locus for age at menarche (locus 19a and 19b in ref. 1); however, the low-frequency variant rs35713889 is poorly tagged by common HapMap2 SNPs (the best proxy rs1134043, $r^{2}=0.24$, was reportedly not associated with age at menarche: $P=0.35$ (ref. 1)). The strongest reported ${ }^{1}$ HapMap 2 signal at this locus is only weakly correlated with rs35713889 (rs3870341, MAF $=26 \%$; $r^{2}=0.07$, distance $422 \mathrm{~kb}$ ), and both signals remained significant when jointly tested in a follow-up sample of 76,831 women from the 23andMe study (in separate models: rs35713889: $\beta=0.08$ years per allele, $P=0.0001$ and rs3870341: $\beta=0.04$, $P=4.5 \times 10^{-5} ;$ in the joint model: rs35713889: $\beta=0.06$, $P=0.004$ and rs3870341: $\beta=0.03, P=0.001)$. LAMB2 encodes one of 15 subunits of Laminin, an extracellular matrix glycoprotein with a key role in the attachment, migration and organization of cells into tissues during embryonic development. Rare recessive mutations in LAMB2 cause Pierson's syndrome (OMIM no. 609049), a disorder characterized by congenital nephrotic syndrome and ocular anomalies, typically with microcoria ${ }^{5}$; neurological abnormalities are also described likely because of cortical laminar disorganization ${ }^{6}$. Common variants in/near other Laminin genes have been reported for a broad range of complex traits, including type 2 diabetes $^{7}$, refractive error ${ }^{8}$, colorectal cancer $^{9}$, IgG glycosylation ${ }^{10}$, ulcerative colitis ${ }^{11}$ and coffee consumption ${ }^{12}$.

A low-frequency missense variant in the TNRC6A gene was associated with later age at menarche (rs113388806; p.Q1112H; MAF $4.7 \% ; \quad \beta=0.08$ years per allele; $P=1.1 \times 10^{-11}$; Supplementary Fig. 3). This signal was only moderately well tagged by common HapMap2 SNPs (best proxy: rs12447003, $r^{2}=0.36$, reported association with age at menarche: $P=0.0005$ (ref. 1)). TNRC6A encodes an Argonaute-navigator protein, responsible for post-transcriptional gene silencing through RNA interference and microRNA pathways ${ }^{13}$. This finding further 


\begin{tabular}{|c|c|c|c|c|c|c|c|c|c|c|c|c|}
\hline \multirow[t]{2}{*}{ Gene } & \multirow[t]{2}{*}{ SNP } & \multirow[t]{2}{*}{ Location } & \multirow[t]{2}{*}{ Alleles ${ }^{\star}$} & \multicolumn{3}{|c|}{ Discovery } & \multicolumn{3}{|c|}{ Follow-up } & \multicolumn{3}{|c|}{ Combined } \\
\hline & & & & Effect (s.e.) & $P$ & $N$ & Effect (s.e.) | VE & $P$ & $\mathbf{N}$ & Effect (s.e.) & $P$ & $N$ \\
\hline \multicolumn{13}{|l|}{ Exome array } \\
\hline ALMS1 & rs45501594 & 2p13.1 & $\mathrm{G} / \mathrm{C} / 1.1 \%$ & $0.26(0.04)$ & $4.6 \mathrm{E}-10$ & 57,867 & $0.23(0.03) \mid 0.12 \%$ & $2.2 \mathrm{E}-11$ & 116,317 & $0.24(0.03)$ & $6.8 \mathrm{E}-20$ & 174,184 \\
\hline LAMB2 & rs35713889 & $3 p 21.31$ & $\mathrm{~T} / \mathrm{C} / 4.4 \%$ & $0.11(0.02)$ & $5.0 \mathrm{E}-07$ & 58,695 & $0.08(0.02) \mid 0.04 \%$ & $2.2 \mathrm{E}-06$ & 116,317 & $0.09(0.01)$ & 1.0E-11 & 175,012 \\
\hline TNRC6A & rs113388806 & $16 \mathrm{p} 12.1$ & $\mathrm{~T} / \mathrm{A} / 4.7 \%$ & $0.09(0.02)$ & $1.7 \mathrm{E}-05$ & 76,657 & $0.08(0.02) \mid 0.04 \%$ & $1.4 \mathrm{E}-07$ & 116,317 & $0.08(0.01)$ & $1.1 \mathrm{E}-11$ & 192,974 \\
\hline TACR3 & rs144292455 & $4 q 24$ & $\mathrm{~T} / \mathrm{C} / 0.08 \%$ & $0.71(0.15)$ & $1.3 \mathrm{E}-06$ & 68,487 & $1.25(0.25) \mid 0.20 \%$ & 8.0E-07 & 116,317 & $0.84(0.13)$ & $2.8 \mathrm{E}-11$ & 184,804 \\
\hline PRKAG1 & rs1126930 & $12 \mathrm{q} 13.12$ & $\mathrm{C} / \mathrm{G} / 3.4 \%$ & $-0.11(0.02)$ & $4.4 \mathrm{E}-07$ & 76,657 & $-0.08(0.02) \mid 0.02 \%$ & $3.6 \mathrm{E}-05$ & 116,317 & $-0.09(0.01)$ & $9.6 \mathrm{E}-11$ & 192,974 \\
\hline \multicolumn{13}{|c|}{ 1000G X-chromosome } \\
\hline IGSF1 & rs762080 & $\mathrm{Xq} 26.2$ & $\mathrm{~A} / \mathrm{C} / 24 \%$ & $-0.07(0.01)$ & $4.1 \mathrm{E}-12$ & 76,831 & $-0.04(0.01) \mid 0.04 \%$ & $6.7 \mathrm{E}-03$ & 39,486 & $-0.06(0.008)$ & $9.4 \mathrm{E}-13$ & 116,317 \\
\hline FAAH2 & rs5914101 & Xp11.21 & $\mathrm{A} / \mathrm{G} / 24 \%$ & $-0.07(0.01)$ & $1.1 \mathrm{E}-09$ & 76,831 & $-0.03(0.01) \mid 0.03 \%$ & $2.0 \mathrm{E}-02$ & 39,486 & $-0.05(0.009)$ & $4.9 \mathrm{E}-10$ & 116,317 \\
\hline
\end{tabular}

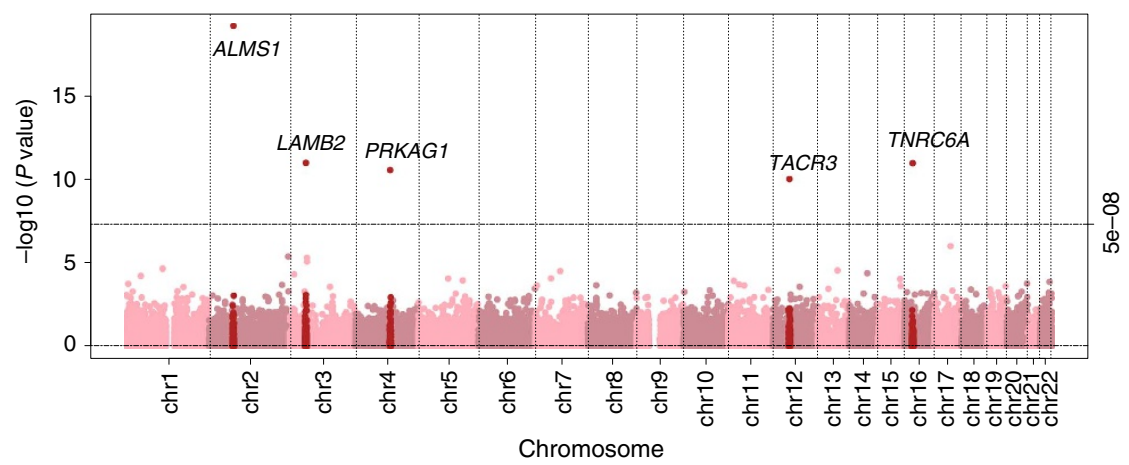

Figure 1 | A 'Manhattan plot' of menarche association statistics for the genotyped low-frequency exome array variants. Test statistics are shown from the exome-chip discovery-phase samples, with the exception of the five labelled loci that indicate results from the combined discovery and replication set.

extends the range of epigenetic mechanisms implicated in the regulation of puberty ${ }^{14}$.

A low-frequency missense variant in PRKAG1 was associated with earlier age at menarche (rs1126930; p.T98S; MAF 3.4\%; $\beta=-0.09$ years per allele, $P=9.6 \times 10^{-11}$; Supplementary Fig. 3). This low-frequency variant is only moderately well tagged by common HapMap2 SNPs $\left(\max r^{2}=0.36\right)$, which reportedly showed subgenome-wide significant association with age at menarche $\left(\mathrm{rs} 11837234, \quad P=3.1 \times 10^{-6}\right)^{1}$. This PRKAG1 missense variant is in the same locus as, but not correlated to, a reported ${ }^{1}$ common signal for age at menarche (rs7138803, $848 \mathrm{~kb}$ apart, $\left.r^{2}=0.02\right)$. PRKAG1 encodes the gamma-1 regulatory subunit of AMP-activated protein kinase, which senses and maintains cellular energy homeostasis by promoting fatty-acid oxidation and inhibiting fatty-acid synthesis; PRKAG1 is overexpressed in ovarian carcinomas ${ }^{15}$ and is somatically mutated in colorectal cancers ${ }^{16}$.

Our second genotyping approach considered X-chromosome GWAS SNPs in up to 76,831 women of European ancestry from the 23 andMe study ${ }^{17}$. Imputation was performed against the 1000 Genomes reference, yielding genotype data for $\sim 266,000$ $\mathrm{X}$-chromosome variants (MAF $>1 \%$ ). Two signals, in/near IGSF1 and $F A A H 2$, reached genome-wide significance for association with age at menarche and both associations were confirmed in 39,486 independent women of European ancestry from the deCODE study.

Common variants in and near IGSF1 were robustly associated with age at menarche (lead SNP: rs762080, MAF $=24 \% ; \beta=0.06$ years per allele, $P=9.4 \times 10^{-13}$; Supplementary Fig. 4). IGSF1 encodes the immunoglobulin superfamily member 1 , which is a plasma membrane glycoprotein highly expressed in the pituitary gland and testis. Rare X-linked mutations in IGSF1 were recently described to cause central hypothyroidism, hypoprolactinemia, delayed puberty and macro-orchidism in males (OMIM no. $300888)^{18,19}$. Heterozygous female carriers reportedly had normal age at menarche; however, 6/18 had central hypothyroidism and $4 / 18$ underwent oophorectomy for ovarian cysts ${ }^{19}$.

The second X-chromosome locus, in Xp11.21 (lead SNP rs5914101 is intronic in FAAH2, MAF $24 \%, \beta=0.05$ years per allele, $P=1.9 \times 10^{-10}$; Supplementary Fig. 4), lies within the critical region for Turner's syndrome, which is the most common cause of primary ovarian insufficiency ${ }^{20}$. FAAH2 encodes fattyacid amide hydrolase 2 . This enzyme catalyses the hydrolysis and degradation of bioactive fatty-acid amides, a large class of endogenous signalling lipids including the endocannabinoids, which modulate several physiological processes, including feeding, inflammation, pain, sleep and various reproductive processes, including hypothalamic gonadotropin-releasing hormone secretion ${ }^{21,22}$.

We sought to further functionally characterize the seven genes implicated by these analyses using expression data on 53 tissue types from the Genotype-Tissue Expression consortium ${ }^{23}$. All seven genes showed high relative tissue expression in the ovary and/or brain (specifically the hypothalamus; Supplementary Fig. 5); however, none of the lead SNPs showed a significant association with mRNA transcript abundance. None of the identified variants were associated with body mass index in 74,071 adults from the deCODE study (all $P>0.05$ ), indicating that their effects on puberty timing are unlikely to be mediated by body mass index.

\section{Discussion}

In summary, by large-scale analysis of genetic variation not captured by previous GWAS for age at menarche, we identified several low-frequency exonic variants of relatively large effect and two common X-chromosome signals. The implicated genes 
provide new insights into the mechanisms that link energy homeostasis to puberty timing, indicate possible roles of RNAmediated gene silencing and fatty-acid amide signalling, and link genes behind rare autosomal, $\mathrm{X}$-linked and syndromic disorders of puberty to normal variation in reproductive timing. Our findings using dense exome arrays in large unselected populations are informative for the clinical interpretation of heterozygous TACR3 variants in patients with rare disorders. In the deCODE study these novel variants collectively explained only $0.5 \%$ of the variance in age at menarche, suggesting that these often overlooked sources of genetic variation do not contribute disproportionately to the missing heritability of this complex trait. While variants with MAF below $1 \%$ are likely not well represented here, our findings indicate that, similar to other complex traits ${ }^{24}$, the genetic architecture of puberty timing is likely dominated by the additive effects of hundreds or even thousands of variants, each with relatively small effect.

\section{Methods}

Exome array discovery analysis. Exome array genotype data were generated across 19 studies in up to 76,657 women of genetically determined European ancestry with questionnaire-reported age at menarche between ages 9 and 17 years (Supplementary Table 1). Exome array genotype calling for three studies (Framingham Heart Study (FHS), the Atherosclerosis Risk in Communities (ARIC) and Rotterdam Study (RS); totalling $\sim 9,000$ women) was performed jointly as part of the CHARGE joint calling protocol ${ }^{25}$, which included over 62,000 individuals. Four additional studies (Cambridge Cancer, KORA, Korcula, Generation Scotland, total $N \sim 9,700$ ) used the cluster file made available by CHARGE to call genotypes. Other studies followed standard calling and QC protocols for the Exome array (Supplementary Table 2). Each contributing study ran a linear regression model on age at menarche, adjusted for birth year and principal components derived from genotypes, using the skatMeta/seqMeta package in R. Studies with family data included a random effect to account for relationships. Alleles were aligned to a common reference file before association testing (SNPInfo_HumanExome-12v1 rev5.tsv.txt available at http://www.chargeconsortium.com/main/exomechip/) and variants with $\mathrm{MAF}>5 \%$ in the meta-analysis were excluded. We performed gene-based testing (within seqMeta) for low-frequency variants using fixed effect burden tests, which assume that all rare variants have the same effect direction and size (scaled by a weight determined by allele frequency), and SKAT tests, which assume that rare variant effects are random and can contain a mixture of null, protective and risk rare alleles. These tests were run using three variant filters, all of which included only variants with $\mathrm{MAF}<1 \%$ : (1) all non-synonymous; (2) non-synonymous annotated as 'damaging' (conserved and predicted damaging, see http://www.chargeconsortium.com/main/exomechip/); and (3) only loss of function. The multiple testing adjustment included two tests $x$ three filters $\times$ number of genes, requiring study-wise significance threshold $P<1.14 \times 10^{-6}$. For individual variants, a fixed-effects inverse variance-weighted meta-analysis was performed across all studies using METAL (http://www.sph.umich.edu/csg/ abecasis/Metal/), with associations considered significant at a conservative genome-wide significance threshold of $P<5 \times 10^{-8}$.

Exome array follow-up studies. We performed follow-up testing of selected exome array variants in the 23andMe study (as described below) and also in 39,486 independent women of European ancestry from the deCODE study, Iceland, who had genotypes on over 34 million variants by imputation of whole-genome sequencing-identified SNPs and indels on Illumina SNP chip data (Supplementary Table 1$)^{26}$. Variants from both studies were required to either pass genotyping QC (23andMe only, described below) or have imputation quality score $>0.4$. $\mathrm{X}$-chromosome follow-up was performed in the deCODE study alone. All participants in all published studies provided informed consent, and the research protocol of each study was approved by their local research ethics committee ${ }^{1}$

$1000 G$ X-chromosome discovery meta-analysis. X-chromosome SNP data were generated in up to 76,831 women of European ancestry from the 23andMe study ${ }^{17,27}$, with questionnaire-reported age at menarche between the ages of 8 and 16 years, and who were genotyped on one or more of three GWAS arrays that also included customized content on human pathogenic variants (Supplementary Table 1$)^{28}$. 23andMe participants provided informed consent to take part in this research under a protocol approved by the AAHRPP-accredited institutional review board, Ethical and Independent Review Services. Before imputation, we excluded SNPs with Hardy-Weinberg equilibrium $P<10^{-20}$, call rate $<95 \%$ or with large allele frequency discrepancies compared with European 1000 Genomes reference data. Frequency discrepancies were identified by computing a $2 \times 2$ table of allele counts for European 1000 Genomes samples and 2,000 randomly sampled
23andMe customers with European ancestry, and identifying SNPs with a $\chi^{2}$ $P<10^{-15}$. Genotype data were imputed against the March 2012 'v3' release of 1000 Genomes reference haplotypes. Age at menarche was assessed by questionnaire and recorded in 2-year-age bins, which were rescaled to 1-year effect estimates post analysis. The validity of this approach was confirmed by the lack of significant heterogeneity between rescaled 23andMe menarche estimates for the 123 previously identified signals and their reported effects ${ }^{1}$. Association results were obtained from linear regression models assuming additive allelic effects. These models included covariates for age and the top five GWAS SNP principal components to account for residual population structure. Results were further adjusted for a lambda genomic control value of 1.152 to correct for any residual test statistic inflation due to population stratification. Linkage disequilibrium score regression analysis (LDSC) ${ }^{29}$ confirmed that principle component correction appropriately controlled for potential test statistic inflation due to population stratification (pre-genomic control-corrected calculated intercept $\sim 1$ ). The reported association test $P$ values were computed from likelihood ratio tests.

X-chromosome follow-up. Identified X-chromosome variants were replicated in 39,486 women from the deCODE study, as described above.

\section{References}

1. Perry, J. R. et al. Parent-of-origin-specific allelic associations among 106 genomic loci for age at menarche. Nature 514, 92-97 (2014).

2. Ozanturk, A. et al. The phenotypic and molecular genetic spectrum of Alstrom syndrome in 44 Turkish kindreds and a literature review of Alstrom syndrome in Turkey. J. Hum. Genet. 60, 1-9 (2014).

3. Collin, G. B. et al. Alms1-disrupted mice recapitulate human Alstrom syndrome. Hum. Mol. Genet. 14, 2323-2333 (2005).

4. Gianetti, E. et al. TAC3/TACR3 mutations reveal preferential activation of gonadotropin-releasing hormone release by neurokinin B in neonatal life followed by reversal in adulthood. J. Clin. Endocrinol. Metab. 95, 2857-2867 (2010).

5. Matejas, V. et al. Mutations in the human laminin beta2 (LAMB2) gene and the associated phenotypic spectrum. Hum. Mutat. 31, 992-1002 (2010).

6. Radner, S. et al. beta2 and gamma3 laminins are critical cortical basement membrane components: ablation of Lamb2 and Lamc3 genes disrupts cortical lamination and produces dysplasia. Dev. Neurobiol. 73, 209-229 (2013).

7. Perry, J. R. et al. Stratifying type 2 diabetes cases by BMI identifies genetic risk variants in LAMA1 and enrichment for risk variants in lean compared to obese cases. PLoS Genet. 8, e1002741 (2012).

8. Verhoeven, V. J. et al. Genome-wide meta-analyses of multiancestry cohorts identify multiple new susceptibility loci for refractive error and myopia. Nat. Genet. 45, 314-318 (2013).

9. Houlston, R. S. et al. Meta-analysis of three genome-wide association studies identifies susceptibility loci for colorectal cancer at 1q41,3q26.2, 12q13.13 and 20q13.33. Nat. Genet. 42, 973-977 (2010).

10. Lauc, G. et al. Loci associated with N-glycosylation of human immunoglobulin $\mathrm{G}$ show pleiotropy with autoimmune diseases and haematological cancers. PLoS Genet. 9, e1003225 (2013).

11. Consortium, U. I. G. et al. Genome-wide association study of ulcerative colitis identifies three new susceptibility loci, including the HNF4A region. Nat. Genet. 41, 1330-1334 (2009).

12. Amin, N. et al. Genome-wide association analysis of coffee drinking suggests association with CYP1A1/CYP1A2 and NRCAM. Mol. Psychiatry. 17, 1116-1129 (2012).

13. Hafner, M. et al. Transcriptome-wide identification of RNA-binding protein and microRNA target sites by PAR-CLIP. Cell 141, 129-141 (2010).

14. Lomniczi, A., Wright, H. \& Ojeda, S. R. Epigenetic regulation of female puberty. Front. Neuroendocrinol. 36, 90-107 (2014).

15. Li, C., Liu, V. W., Chiu, P. M., Chan, D. W. \& Ngan, H. Y. Over-expressions of AMPK subunits in ovarian carcinomas with significant clinical implications. BMC Cancer 12, 357 (2012).

16. Seshagiri, S. et al. Recurrent R-spondin fusions in colon cancer. Nature 488, 660-664 (2012).

17. Hinds, D. A. et al. A genome-wide association meta-analysis of self-reported allergy identifies shared and allergy-specific susceptibility loci. Nat. Genet. 45, 907-911 (2013).

18. Sun, Y. et al. Loss-of-function mutations in IGSF1 cause an X-linked syndrome of central hypothyroidism and testicular enlargement. Nat. Genet. 44, 1375-1381 (2012).

19. Joustra, S. D. et al. The IGSF1 deficiency syndrome: characteristics of male and female patients. J. Clin. Endocrinol. Metab. 98, 4942-4952 (2013).

20. Zinn, A. R. et al. Evidence for a Turner syndrome locus or loci at Xp11.2-p22.1. Am. J. Hum. Genet. 63, 1757-1766 (1998).

21. Gammon, C. M., Freeman, Jr G. M., Xie, W., Petersen, S. L. \& Wetsel, W. C Regulation of gonadotropin-releasing hormone secretion by cannabinoids. Endocrinology 146, 4491-4499 (2005). 
22. Meccariello, R., Battista, N., Bradshaw, H. B. \& Wang, H. Updates in reproduction coming from the endocannabinoid system. Int. J. Endocrinol. 2014, 412354 (2014).

23. Consortium, G. T.. The Genotype-Tissue Expression (GTEx) project. Nat. Genet. 45, 580-585 (2013).

24. Wood, A. R. et al. Defining the role of common variation in the genomic and biological architecture of adult human height. Nat. Genet. 46, 1173-1186 (2014).

25. Grove, M. L. et al. Best practices and joint calling of the HumanExome BeadChip: the CHARGE Consortium. PLoS ONE 8, e68095 (2013).

26. Steinthorsdottir, V. et al. Identification of low-frequency and rare sequence variants associated with elevated or reduced risk of type 2 diabetes. Nat. Genet. 46, 294-298 (2014)

27. Tung, J. Y. et al. Efficient replication of over 180 genetic associations with self-reported medical data. PLoS ONE 6, e23473 (2011).

28. Eriksson, N. et al. Web-based, participant-driven studies yield novel genetic associations for common traits. PLoS Genet. 6, e1000993 (2010).

29. Bulik-Sullivan, B. K. et al. LD Score regression distinguishes confounding from polygenicity in genome-wide association studies. Nat. Genet. 47, 291-295 (2015).

\section{Acknowledgements}

Sources of funding for the contributing studies are listed in Supplementary Table 3.

\section{Author contributions}

All authors reviewed the original and revised manuscripts. Analysis: K.L.L., F.R.D., P.S., K.S.R., T.E., C.E.E., E.A., C.H., J.E.H., E.M., E.P., A.R., L.M.R., U.M.S., L.S., A.T., D.J.T., M.T., C.A.W., L.M.Y.-A. and J.R.B.P.; sample collection, phenotyping and genotyping:
A.C.A., C.B., A.D.C., F.C., E.W.D., A.M.D., I.G., M.L.G., D.F.G., L.J.H., A.H., J.H., R.D.J., D.K., J.K., E.M.L., L.A.L., C.L., X.L., J.L., R.M., A.C.M., S.P., A.P., O.P., D.P., A.P.R., F.R., I.R., C.F.S., D.S., R.A.S., D.St., J.A.V., U.V., D.V., J.G.W and M.Z; study PI: E.B., J.E.B., L.C., D.F.E., C.Ha., F.B.H., S.L., A.M., C.E.P., P.M.R., K.S., E.A.S., D.T., A.G.U., S.U., H.V., N.J.W., M.W., N.F., D.I.C., U.T., A.Mu., Ka.S., J.M.M. and K.K.O.; working group/project management: K.L.L., F.R.D., P.S., K.S.R., T.E., C.E.E., N.F., D.I.C., U.T., A.Mu., Ka.S., J.M.M., K.K.O and J.R.B.P.

\section{Additional information}

Supplementary Information accompanies this paper at http://www.nature.com/ naturecommunications

Competing financial interests: The authors declare no competing financial interests.

Reprints and permission information is available online at http://npg.nature.com/ reprintsandpermissions/

How to cite this article: Lunetta, K. L. et al. Rare coding variants and X-linked loci associated with age at menarche. Nat. Commun. 6:7756 doi: 10.1038/ncomms8756 (2015).

This work is licensed under a Creative Commons Attribution 4.0 International License. The images or other third party material in this article are included in the article's Creative Commons license, unless indicated otherwise in the credit line; if the material is not included under the Creative Commons license, users will need to obtain permission from the license holder to reproduce the material To view a copy of this license, visit http://creativecommons.org/licenses/by/4.0/

Kathryn L. Lunetta ${ }^{1,2, \star}$, Felix R. Day ${ }^{3, \star}$, Patrick Sulem ${ }^{4, \star}$, Katherine S. Ruth ${ }^{5}$, Joyce Y. Tung ${ }^{6}$, David A. Hinds ${ }^{6}$, Tõnu Esko ${ }^{7,8,9,10}$, Cathy E. Elks ${ }^{3}$, Elisabeth Altmaier ${ }^{11,12}$, Chunyan He ${ }^{13,14}$, Jennifer E. Huffman ${ }^{15}$, Evelin Mihailov ${ }^{7}$, Eleonora Porcu 16,17,18, Antonietta Robino ${ }^{19}$, Lynda M. Rose ${ }^{20}$, Ursula M. Schick ${ }^{21}$, Lisette Stolk22, Alexander Teumer ${ }^{23}$, Deborah J. Thompson ${ }^{24}$, Michela Traglia25, Carol A. Wang26, Laura M. Yerges-Armstrong ${ }^{27}$, Antonis C. Antoniou ${ }^{24}$, Caterina Barbieri ${ }^{25}$, Andrea D. Coviello ${ }^{28}$, Francesco Cucca ${ }^{16,17}$, Ellen W. Demerath ${ }^{29}$, Alison M. Dunning ${ }^{30}$, llaria Gandin ${ }^{19,31}$, Megan L. Grove ${ }^{32}$, Daniel F. Gudbjartsson 4,33, Lynne J. Hocking ${ }^{34}$, Albert Hofman ${ }^{35}$, Jinyan Huang ${ }^{36}$, Rebecca D. Jackson ${ }^{37}$, David Karasik ${ }^{38,39}$, Jennifer Kriebel ${ }^{11,40}$, Ethan M. Lange ${ }^{41,42}$, Leslie A. Lange ${ }^{41}$, Claudia Langenberg ${ }^{3}$, Xin Li43, Jian'an Luan ${ }^{3}$, Reedik Mägi ${ }^{7}$, Alanna C. Morrison ${ }^{32}$, Sandosh Padmanabhan ${ }^{44}$, Ailith Pirie ${ }^{24}$, Ozren Polasek ${ }^{45}$, David Porteous ${ }^{46}$, Alex P. Reiner21, Fernando Rivadeneira ${ }^{22,35}$, Igor Rudan ${ }^{47}$, Cinzia F. Sala ${ }^{25}$, David Schlessinger ${ }^{48}$, Robert A. Scott ${ }^{3}$, Doris Stöckl ${ }^{49}$, Jenny A. Visser ${ }^{22}$, Uwe Völker ${ }^{50}$, Diego Vozzi ${ }^{19}$, James G. Wilson ${ }^{51}$, Marek Zygmunt ${ }^{52}$, EPIC-InterAct Consortium ${ }^{\dagger}$, Generation Scotland ${ }^{\dagger}$, Eric Boerwinkle ${ }^{32}$, Julie E. Buring ${ }^{20,39}$, Laura Crisponi ${ }^{16}$, Douglas F. Easton ${ }^{24,30}$, Caroline Hayward ${ }^{15}$, Frank B. Hu ${ }^{43,53,54}$, Simin Liu ${ }^{55}$, Andres Metspalu ${ }^{7,56}$, Craig E. Pennell ${ }^{26}$, Paul M. Ridker ${ }^{20,39}$, Konstantin Strauch ${ }^{12,57}$, Elizabeth A. Streeten ${ }^{27}$, Daniela Toniolo ${ }^{25}$, André G. Uitterlinden ${ }^{22,35}$, Sheila Ulivi ${ }^{19}$, Henry Völzke ${ }^{23}$, Nicholas J. Wareham ${ }^{3}$, Melissa Wellons ${ }^{58}$, Nora Franceschini ${ }^{59}$, Daniel I. Chasman ${ }^{20,39}$, Unnur Thorsteinsdottir ${ }^{4,60}$, Anna Murray ${ }^{5}$, Kari Stefansson ${ }^{4,60}$, Joanne M. Murabito ${ }^{2,61}$, Ken K. Ong ${ }^{3,62, \star} \&$ John R.B. Perry ${ }^{3, \star}$

\footnotetext{
${ }^{1}$ Boston University School of Public Health, Department of Biostatistics, Boston, Massachusetts 02118, USA. ${ }^{2}$ NHLBI's and Boston University's Framingham Heart Study, Framingham, Massachusetts 01702-5827, USA. ${ }^{3}$ MRC Epidemiology Unit, University of Cambridge School of Clinical Medicine, Box 285 Institute of Metabolic Science, Cambridge Biomedical Campus, Cambridge CB2 OQQ, UK. ${ }^{4}$ deCODE genetics/Amgen, Inc., Reykjavik IS-101, Iceland.

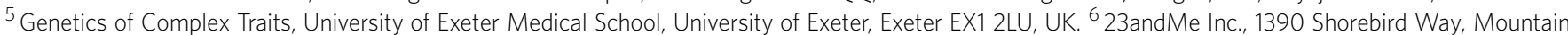
View, California 94043, USA. ${ }^{7}$ Estonian Genome Center, University of Tartu, Tartu 51010, Estonia. ${ }^{8}$ Division of Endocrinology, Boston Children's Hospital, Boston, MA 02115, USA. ${ }^{9}$ Department of Genetics, Harvard Medical School, Boston, MA 02115, USA. ${ }^{10}$ Broad Institute of the Massachusetts Institute of Technology and Harvard University, 140, Cambridge, MA 02142, USA. ${ }^{11}$ Research Unit of Molecular Epidemiology, Helmholtz Zentrum München-German Research Center for Environmental Health, Neuherberg 85764, Germany. ${ }^{12}$ Institute of Genetic Epidemiology, Helmholtz Zentrum München-German Research Center for Environmental Health, Neuherberg 85764, Germany. ${ }^{13}$ Department of Epidemiology, Indiana University Richard M. Fairbanks School of Public Health, Indianapolis, IN 46202, USA. ${ }^{14}$ Indiana University Melvin and Bren Simon Cancer Center, Indianapolis, IN 46202, USA. ${ }^{15}$ Medical Research Council Human Genetics Unit, Institute of Genetics and Molecular Medicine, University of Edinburgh, Edinburgh EH4 2XU, UK. ${ }^{16}$ Institute of Genetics and
} 
Biomedical Research, National Research Council, Cagliari, Sardinia 09042, Italy. ${ }^{17}$ University of Sassari, Department of Biomedical Sciences, Sassari, Sassari 07100, Italy. ${ }^{18}$ Center for Statistical Genetics, Ann Arbor, University of Michigan, Michigan 48109-2029, USA. 19 Institute for Maternal and Child HealthIRCCS "Burlo Garofolo", Trieste 34137, Italy. ${ }^{20}$ Division of Preventive Medicine, Brigham and Women's Hospital, Boston, MA $02215 .{ }^{21}$ Fred Hutchinson Cancer Research Center, Public Health Sciences Division, Seattle, WA 98109-1024, USA. ${ }^{22}$ Department of Internal Medicine, Erasmus MC, Rotterdam 3015GE, the Netherlands. ${ }^{23}$ Institute for Community Medicine, University Medicine Greifswald, Greifswald 17475, Germany. ${ }^{24}$ Centre for Cancer Genetic Epidemiology, Department of Public Health and Primary Care, University of Cambridge, CB1 8RN, UK. ${ }^{25}$ Division of Genetics and Cell Biology, San Raffaele Scientific Institute, Milano 20132, Italy. ${ }^{26}$ School of Women's and Infants' Health, The University of Western Australia, WA-6009, Australia. ${ }^{27}$ Program in Personalized Medicine, Division of Endocrinology, Diabetes and Nutrition-University of Maryland School of Medicine, Baltimore, MD 21201, USA. ${ }^{28}$ Boston University School of Medicine, Department of Medicine, Sections of Preventive Medicine and Endocrinology, Boston, MA, USA. ${ }^{29}$ Division of Epidemiology \& Community Health, University of Minnesotta, Minneapolis, MN 55455, USA. ${ }^{30}$ Centre for Cancer Genetic Epidemiology, Department of Oncology, University of Cambridge, Cambridge CB1 8RN, UK. ${ }^{31}$ Department of Clinical Medical Sciences, Surgical and Health, University of Trieste, Trieste 34149 , Italy. ${ }^{32}$ Human Genetics Center, School of Public Health, The University of Texas Health Science Center at Houston, Houston, TX 77030, USA. ${ }^{33}$ School of Engineering and Natural Sciences, University of Iceland, Reykjavik IS-101, Iceland. ${ }^{34}$ Musculoskeletal Research Programme, Division of Applied Medicine, University of Aberdeen, Aberdeen AB25 2ZD, UK. ${ }^{35}$ Genetic Epidemiology Unit Department of Epidemiology, Erasmus MC, Rotterdam 3015 GE, the Netherlands. ${ }^{36}$ State Key Laboratory of Medical Genomics, Shanghai Institute of Hematology, Rui Jin Hospital, Shanghai Jiao Tong University School of Medicine, Shanghai 200025, China. ${ }^{37}$ Department of Internal Medicine, The Ohio State University, Columbus, Ohio 43210, USA. ${ }^{38}$ Hebrew SeniorLife Institute for Aging Research, Boston, MA 02131, USA. ${ }^{39}$ Harvard Medical School, Boston, MA 02115, USA. ${ }^{40}$ German Center for Diabetes Research, Neuherberg 85764 , Germany. ${ }^{41}$ Department of Genetics, University of North Carolina, Chapel Hill, NC 27599, USA. ${ }^{42}$ Department of Biostatistics, University of North Carolina, Chapel Hill, NC 27599, USA. ${ }^{43}$ Department of Epidemiology, Harvard School of Public Health, Boston, MA 02115, USA. ${ }^{44}$ British Heart Foundation Glasgow Cardiovascular Research Centre, Institute of Cardiovascular and Medical Sciences, College of Medical, Veterinary and Life Sciences, University of Glasgow, Glasgow G12 8TA, UK. ${ }^{45}$ Faculty of Medicine, University of Split, Split, Croatia. ${ }^{46}$ Medical Genetics Section, Centre for Genomic and Experimental Medicine, Institute of Genetics and Molecular Medicine, University of Edinburgh, Edinburgh EH4 2XU, UK. ${ }^{47}$ Institute for Population Health Sciences and Informatics, University of Edinburgh, Teviot Place, Edinburgh EH8 9AG, Scotland. ${ }^{48}$ National Institute on Aging, Intramural Research Program, Baltimore, MD 20892, USA. ${ }^{49}$ Institute of Epidemiology II, Helmholtz Zentrum München - German Research Center for Environmental Health, Neuherberg 85764 , Germany. 50 Interfaculty Institute for Genetics and Functional Genomics, University Medicine Greifswald, Greifswald 17475, Germany. ${ }^{51}$ Department of Physiology and Biophysics, University of Mississippi Medical Center, Jackson, MS 39216, USA. ${ }^{52}$ Department of Obstetrics and Gynecology, University Medicine Greifswald, Greifswald 17475, Germany. ${ }^{53}$ Channing Division of Network Medicine, Department of Medicine, Brigham and Women's Hospital and Harvard Medical School, Boston, MA 02115, USA. ${ }^{54}$ Department of Nutrition, Harvard School of Public Health, Boston, MA 02115, USA. ${ }^{55}$ Departments of Epidemiology and Medicine Brown University, Brown University, Providence, RI 02912, USA. ${ }^{56}$ Institute of Molecular and Cell Biology, University of Tartu, Tartu 51010, Estonia. 57 Institute of Medical Informatics, Biometry and Epidemiology, Chair of Genetic Epidemiology, Ludwig-Maximilians-Universität, Munich 81377, Germany. 58 Department of Medicine, Vanderbilt University Medical Center, Nashville, Tennessee 37203, USA. ${ }^{59}$ Department of Epidemiology, University of North Carolina, Chapel Hill, NC 27599, USA. ${ }^{60}$ Faculty of Medicine, University of Iceland, Reykjavik IS-101, Iceland. ${ }^{61}$ Boston University School of Medicine, Department of Medicine, Section of General Internal Medicine, Boston, MA 02118, USA. ${ }^{62}$ Department of Paediatrics, University of Cambridge, Cambridge $\mathrm{CB} 2 \mathrm{OQQ}, \mathrm{UK}$. * These authors contributed equally to this work. †A full list of consortium members appears at the end of the paper.

\section{EPIC-InterAct Consortium}

Nita G. Forouhi' ${ }^{3}$, Nicola D. Kerrison ${ }^{3}$, Stephen J. Sharp³, Matt Sims 3 , Inês Barroso 63,64 , Panos Deloukas ${ }^{63}$, Mark I. McCarthy 65,66,67, Larraitz Arriola $68,69,70$, Beverley Balkau71772, Aurelio Barricarte ${ }^{70,73}$, Heiner Boeing 74 , Paul W. Franks ${ }^{75,76}$, Carlos Gonzalez ${ }^{77}$, Sara Grioni ${ }^{78}$, Rudolf Kaaks ${ }^{79}$, Timothy J. Key ${ }^{80}$, Carmen Navarro ${ }^{70,81,82}$, Peter M. Nilsson ${ }^{75}$, Kim Overvad ${ }^{83,84}$, Domenico Palli ${ }^{85}$, Salvatore Panico ${ }^{86}$, J. Ramón Quirós ${ }^{87}$, Olov Rolandsson ${ }^{3,76}$, Carlotta Sacerdote ${ }^{88,89}$, María-José Sánchez ${ }^{70,90,91}$, Nadia Slimani ${ }^{92}$, Anne Tjonneland ${ }^{93}$, Rosario Tumino 94,95 , Daphne L. van der A 96 , Yvonne T. van der Schouw ${ }^{97}$ \& Elio Riboli 98

${ }^{63}$ The Wellcome Trust Sanger Institute, Cambridge, CB10 1SA, UK. ${ }^{64}$ University of Cambridge Metabolic Research Laboratories, Cambridge CB2 0QQ, UK. ${ }^{65}$ Oxford Centre for Diabetes, Endocrinology and Metabolism (OCDEM), University of Oxford, OX3 7LJ, UK. 66 Wellcome Trust Centre for Human Genetics, University of Oxford, Oxford OX3 7BN, UK. ${ }^{67}$ Oxford NIHR Biomedical Research Centre, Oxford OX3 7LJ, UK. ${ }^{68}$ Public Health Division of Gipuzkoa, 20013 San Sebastian, Spain. ${ }^{69}$ Instituto BIO-Donostia, Basque Government, 20014 Donostia, San Sebastian, Spain. ${ }^{70}$ CIBER Epidemiología y Salud Pública (CIBERESP), 28029 Madrid, Spain. ${ }^{71}$ Inserm, CESP, U1018, 94807 Villejuif cedex, France. ${ }^{72}$ Univ Paris-Sud, UMRS 1018, F-94805, Villejuif, France.

${ }^{73}$ Navarre Public Health Institute (ISPN), 31003 Pamplona, Spain. ${ }^{74}$ German Institute of Human Nutrition Potsdam-Rehbruecke, 14558 Nuthetal Germany. ${ }^{75}$ Department of Clinical Sciences, Lund University, S-205 02 Malmö, Sweden. ${ }^{76}$ Department of Public Health and Clinical Medicine, Umeå University, 90187 Umeå, Sweden. ${ }^{77}$ Catalan Institute of Oncology (ICO), 08916 Badalona, Barcelona, Spain. ${ }^{78}$ Epidemiology and Prevention Unit, 20133 Milan, Italy. ${ }^{79}$ German Cancer Research Centre (DKFZ), 69120 Heidelberg, Germany. ${ }^{80}$ Cancer Epidemiology Unit, Nuffield Department of Population Health, University of Oxford, OX3 7LF, UK. ${ }^{81}$ Department of Epidemiology, Murcia Regional Health Council, 30008 Murcia, Spain. ${ }^{82}$ Unit of Preventive Medicine and Public Health, School of Medicine, University of Murcia, 30100 Espinardo, Spain. ${ }^{83}$ Department of Public Health, Section for Epidemiology, Aarhus University,

DK-8000 Aarhus C, Denmark. ${ }^{84}$ Aalborg University Hospital, 9100 Aalborg, Denmark. ${ }^{85}$ Cancer Research and Prevention Institute (ISPO), 50141 Florence,

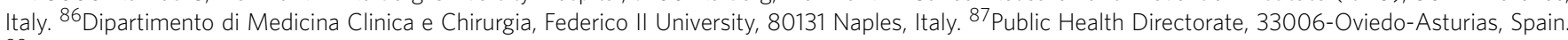
${ }^{88}$ Unit of Cancer Epidemiology, Citta' della Salute e della Scienza Hospital-University of Turin and Center for Cancer Prevention (CPO), 10126 Torino, Italy. ${ }^{89}$ Human Genetics Foundation (HuGeF), 10126 Torino, Italy. ${ }^{90}$ Andalusian School of Public Health, 18080 Granada, Spain. ${ }^{91}$ Instituto de Investigaciœn Biosanitaria de Granada (Granada.ibs), 18012 Granada, Spain. ${ }^{92}$ International Agency for Research on Cancer, 69372 Lyon Cedex 08 , France. ${ }^{93}$ Danish Cancer Society Research Center, 2100 Copenhagen, Denmark. ${ }^{94}$ ASP, 97100 Ragusa, Italy. ${ }^{95}$ Ragusa Cancer Registry, Aire Onlus, 97100 Ragusa, Italy. ${ }^{96}$ National Institute for Public Health and the Environment (RIVM), 3720 BA Bilthoven, The Netherlands. ${ }^{97}$ University Medical Center Utrecht, 3508 GA Utrecht, the Netherlands. ${ }^{98}$ School of Public Health, Imperial College London, W2 1PG, UK. 


\section{Generation Scotland}

Blair H. Smith 46,99, Archie Campbell 99 , Ian J. Deary99,100,101, Andrew M. Mclntosh99,102

${ }^{99}$ Centre for Genomic and Experimental Medicine, Institute of Genetics and Molecular Medicine, University of Edinburgh, Edinburgh EH4 2XU, UK.

${ }^{100}$ Centre for Cognitive Ageing and Cognitive Epidemiology, University of Edinburgh, Edinburgh, UK. ${ }^{101}$ Department of Psychology, University of Edinburgh, EH8 9JZ, UK. ${ }^{102}$ Division of Psychiatry, University of Edinburgh, Edinburgh, EH10 5HF, UK. 


\section{Corrigendum: Rare coding variants and X-linked loci associated with age at menarche}

Kathryn L. Lunetta, Felix R. Day, Patrick Sulem, Katherine S. Ruth, Joyce Y. Tung, David A. Hinds, Tõnu Esko, Cathy E. Elks, Elisabeth Altmaier, Chunyan He, Jennifer E. Huffman, Evelin Mihailov, Eleonora Porcu, Antonietta Robino, Lynda M. Rose, Ursula M. Schick, Lisette Stolk, Alexander Teumer, Deborah J. Thompson, Michela Traglia, Carol A. Wang, Laura M. Yerges-Armstrong, Antonis C. Antoniou, Caterina Barbieri, Andrea D. Coviello, Francesco Cucca, Ellen W. Demerath, Alison M. Dunning, Ilaria Gandin, Megan L. Grove, Daniel F. Gudbjartsson, Lynne J. Hocking, Albert Hofman, Jinyan Huang, Rebecca D. Jackson,

David Karasik, Jennifer Kriebel, Ethan M. Lange, Leslie A. Lange, Claudia Langenberg, Xin Li, Jian'an Luan, Reedik Mägi, Alanna C. Morrison, Sandosh Padmanabhan, Ailith Pirie, Ozren Polasek, David Porteous, Alex P. Reiner, Fernando Rivadeneira, Igor Rudan, Cinzia F. Sala, David Schlessinger, Robert A. Scott, Doris Stöckl, Jenny A. Visser, Uwe Völker, Diego Vozzi, James G. Wilson, Marek Zygmunt, EPIC-InterAct Consortium, Generation Scotland, Eric Boerwinkle, Julie E. Buring, Laura Crisponi, Douglas F. Easton, Caroline Hayward, Frank B. Hu, Simin Liu, Andres Metspalu, Craig E. Pennell, Paul M. Ridker, Konstantin Strauch, Elizabeth A. Streeten, Daniela Toniolo, André G. Uitterlinden, Sheila Ulivi, Henry Völzke, Nicholas J. Wareham, Melissa Wellons, Nora Franceschini, Daniel I. Chasman, Unnur Thorsteinsdottir, Anna Murray, Kari Stefansson, Joanne M. Murabito, Ken K. Ong \& John R.B. Perry

Nature Communications 6:7756 doi: 10.1038/ncomms8756 (2015); Published 4 Aug 2015; Updated 17 Dec 2015

In the Results section and in the legend of Table 1 of this Article, the company deCODE genetics, Inc. is incorrectly referred to as 'Diabetes Epidemiology: collaborative analysis of Diagnostic criteria in Europe'. In neither instance is deCODE an abbreviation.

\footnotetext{
(c) (i) This work is licensed under a Creative Commons Attribution 4.0 International License. The images or other third party material in this article are included in the article's Creative Commons license, unless indicated otherwise in the credit line; if the material is not included under the Creative Commons license, users will need to obtain permission from the license holder to reproduce the material. To view a copy of this license, visit http://creativecommons.org/licenses/by/4.0/
} 